\title{
Pesticide Safety Around Animals ${ }^{1}$
}

\author{
P. E. Kaufman and E. N. I. Weeks ${ }^{2}$
}

Arthropod pests of domestic animals are a serious threat to an animal's health because many feed on body tissues such as blood, skin, hair or feathers, and in wounds. Skin irritation produced by these pests feeding often results in discomfort for the animal. Many pests are also vectors of disease-causing pathogens. In general, infested animals are unhealthy and cannot be managed efficiently.

Pesticides are commonly used to protect animals from pests. However, it is important to remember that any pesticide should be considered an active poison. The successful control of pests requires careful mixing and application of recommended pesticides according to label directions. Besides ensuring the control of pests, applying pesticides at the recommended rate is necessary to prevent injury to the animal. The application rate on the label was selected on the basis of experiments, and thus the dose that is listed on the label is both safe to use and adequate to control pests. Therefore, the directions on the label should be followed exactly. Never use more insecticide than is recommended on the label. Using less insecticide than what is recommended is also not advised, because it can make pests resistant to the active compounds in the pesticide. Pesticides will be less effective against resistant pests. It is illegal to mix and apply pesticide except in the manner specified on the label; violators can be fined.

\section{Selecting Pesticides}

Pesticides should be carefully selected after reviewing the recommendations made by extension personnel and carefully reading the labels of pesticides. Before you purchase a pesticide, read the label to determine whether:

1. The label lists the name of the pest you plan to control.

2. The animal or site on which you plan to use the pesticide is listed.

3. The formulation is suitable for the job.

4. The right application equipment is available.

The proper safety equipment and personal protective equipment is available.

Also read the label to determine the amount of pesticide needed and the restrictions on its use.

Do not purchase more than you will use.

\section{Storage of Pesticides}

Pesticides should be stored in a locked, well-ventilated, dry room away from food or feed, and out of reach of livestock, pets, children and wild animals. The storage room should be located away from buildings where people live or where animals are kept, and a sign should be posted near the entrance warning that poisons are inside. Construction should be of fire-resistant materials with insulation to

1. This document is ENY-272, one of a series of the Entomology and Nematology Department, Florida Cooperative Extension Service, Institute of Food and Agricultural Sciences, University of Florida. Original publication date May 1995. Reviewed April 2012. Revised October 2012. Visit the EDIS website at http://edis.ifas.ufl.edu.

2. P. E. Kaufman, associate professor/Extension entomologist, Entomology and Nematology Department; and E. N. I. Weeks, assistant research scientist, Entomology and Nematology Department, Cooperative Extension Service, Institute of Food and Agricultural Sciences, University of Florida, Gainesville, FL 32611 . First published in May 1995 by J. A. Hogsette, USDA Laboratory, Gainesville, FL; P. G. Koehler, professor/Extension entomologist; and P. E. Kaufman, assistant professor/extension entomologist, Entomology and Nematology Department. 
prevent freezing and overheating. An exhaust fan should be installed in one wall at the most distant point from the door and designed to operate only when the door is open. The fan will remove pesticide fumes and keep fresh air flowing through the storage room while it is occupied.

Pesticides should be stored in the original containers and checked periodically for leaks. Leaking containers should be placed in "secondary containment," which means put the leaking container inside of a larger container. Do not store herbicides and insecticides together because contamination can occur. Clean up spills promptly and properly. Keep a suitable spill kit near any stored pesticides. For example, sawdust or absorbent cat litter, a shovel, a watertight container and personal protective equipment like coveralls, boots, gloves, goggles and a respirator. Store empty pesticide containers in the pesticide storage room until they can be disposed of as directed on the pesticide label. Keep an up-to-date inventory of the pesticides in the storage room.

\section{Pesticide Application}

Before applying the pesticide, read the label again and look for the following additional information:

1. The target pest

2. The animal or area to be treated

3. The necessary personal protective equipment

4. Special warnings and first aid measures

5. Correct mixing procedures

6. Rate of application

7. Restrictions on use

Never use a higher rate of pesticide than is stated on the label. Overdoses of pesticides can kill animals or weaken them and make them susceptible to disease. Young animals, because of their low body weight, are particularly susceptible to overdoses of some pesticides.

Precautions should be taken during mixing, application and equipment clean-up to insure the safety of the animal. These are:

1. Cover feed and water containers to prevent contamination during application.

2. Keep chemicals thoroughly mixed in sprayer. Some formulations will separate unless constantly agitated, resulting in overdoses for some animals and under doses for others.

3. Do not mix pesticides or load a sprayer where animals (including children, pets, livestock or wild animals) may have access to spilled chemicals.
4. Do not use a pesticide if an oily or gummy film is present on the surface. The chemical may have deteriorated in storage.

5. Pesticides should not be applied to animals already treated with other pesticides or drugs. The combination of chemicals may produce undesirable effects.

6. Sick or stressed animals should not be treated with certain pesticides.

7. Young animals are often susceptible to pesticides. Read label precautions before treating young animals.

8. Do not use sprayers with leaking tanks, hoses or connections. Leaking pesticide solution increases the risk for contamination of humans, livestock, and premises and the chance of under/overdosing.

9. Certain types of animals are susceptible to some pesticides. Sensitive animals should not be treated. (See Problems with Pesticides below).

10. Some solvents tend to stay in the air and cause toxicity problems to animals while in the holding area. To prevent this problem, animals should not be treated during hot, still afternoons.

11. Have a dedicated area for cleaning sprayers so that excess pesticide and wash water do not contaminate animals or food and water. Keep drainage out of water supplies and streams. Do not allow animals to come into areas where sprayers are cleaned.

12. Mix only the amount of chemical needed for the number of animals to be treated at that time.

13. Excess pesticide solutions left in spray tanks should be disposed of properly. Do not store pesticides after they have been mixed for use except where permitted by label instructions. Many pesticides lose potency when mixed for use and then stored. In addition to the above precautions, protect yourself from the effects of pesticides by following all safety procedures on the label and using accepted pest control practices.

\section{Pesticide Residues}

Many animals are raised for human consumption. At slaughter, these animals must not contain potentially dangerous amounts of pesticides in the edible tissues. The Federal Government through the Food and Drug Administration protects us from food with harmful pesticide residues by establishing pesticide tolerance levels. A pesticide tolerance level is the amount of pesticide that may remain as a residue in food. For example, the tolerance level for chlorinated hydrocarbons in meat, eggs and milk is zero. It is important that illegal levels of pesticide residues do not accumulate in edible tissues. If a food product containing amounts of pesticide greater than the established tolerance level is found by the Food and Drug Administration, the 
product may be seized and destroyed. Legal action may also be taken against persons and businesses responsible for the products with high-level residues.

To prevent a build-up of high-level residues, observe the following precautions:

1. Use only approved pesticides at the recommended dosage.

2. After application of a pesticide, allow the prescribed number of days to elapse (read the pesticide label) before slaughtering the animal or using edible animal products such as milk or eggs. The interval will permit the elimination of pesticide from the animal's tissues prior to processing of edible commodities.

3. When preparing backrubbers, use only recommended materials and carriers. Do not use waste motor oil or oil of unknown origin because it may contain materials that could produce undesirable residues. See ENY-279, "Self-treatment Methods for Livestock-Backrubbers," for more information about the safe use of backrubbers.

4. Pastures, forages, and feed crops should only be treated with pesticides that will not contaminate the crop.

Allow the prescribed number of days to elapse between pesticide application and crop harvest or use.

5. Clean application equipment thoroughly so that residues from previously used chemicals are not unknowingly applied to animals.

6. Do not give animals feed or food of unknown origin unless you are sure it will not cause illegal residue problems.

7. Do not allow pesticides being applied in nearby areas to drift onto animals or into areas frequented by animals.

8. Do not allow feed or water to become contaminated with pesticides.

9. Do not make repeat applications unless specified on the label.

\section{Problems with Pesticides}

Applicators should be aware that certain animal breeds may react adversely to certain pesticides or materials contained in pesticide formulations. Sensitive animals should not be treated or should be treated with extreme care. For instance, Brahman cattle are sensitive to some organophosphate pesticides. If a pesticide label states "Do not treat Brahman cattle," then the pesticide should never be applied to those animals. Also the skin of some horses and dogs is sensitive to certain pesticide formulations. If you are uncertain about an animal's sensitivity, treat a small area of skin and observe the area for 24 hours before treating the entire animal.
Individual animals may also react adversely to a pesticide or pesticide formulation. Sensitive animals should be identified and treated only with acceptable chemicals. If an animal does react adversely to an application, remove the pesticide formulation from the animal by drenching the skin with water and then following the advice given below for specific pesticide types. In general, the animal should be washed with water (room temperature) and detergent. The animal should not be scrubbed because increased blood circulation and abrasion could enhance absorption of the pesticide.

The age, size and condition of an animal are important when applying pesticides. Young animals are usually smaller than mature animals and consequently more susceptible to pesticides. Take care when treating young animals, and check the label to see whether precautions warn against application to young animals. Many insecticides should be applied according to the size of the animal. Generally speaking, less pesticide should be applied to smaller animals to prevent toxicity problems. Dusts and wettable powder formulations are usually less toxic than emulsifiable formulations and easier to remove should problems develop. Stressed or diseased animals are more sensitive to pesticides. Often the additional stress of a pesticide application is enough to kill an unhealthy animal.

Certain precautions can be taken during routine applications to prevent pesticide problems. These are:

1. Do not apply pesticides on extremely hot, still days, when chances for toxicity problems are increased.

2. Do not wet animals with spray or dip during cold or freezing weather.

3. Use dust formulations whenever possible because they are absorbed more slowly through the skin than liquid formulations and are, therefore, usually less toxic. Dusts also do not add excessive moisture to the animal. For sensitive animals, use dust or wettable powders.

4. Do not treat stressed, diseased, young, or small animals without consulting the pesticide label.

5. Do not overdose animals by treating them at the same time with several different pesticides aimed at different target pests. Choose one pesticide that most effectively controls all pests involved, or treat each pest individually at different times. 


\section{Pesticide Toxicity}

Following the elimination or restriction of the use of many chlorinated hydrocarbon pesticides, the use of organophosphate and carbamate pesticides for pest control on or around animals increased. In general, organophosphate and carbamate pesticides are more toxic to warm-blooded animals and active for shorter periods of time than chlorinated hydrocarbons. Consequently, more toxic compounds were being applied more often, which resulted in increased incidences of pesticide poisonings among domestic and agricultural animals.

Through the re-registration process under the Food Quality Protection Act of 1996, many organophosphate and carbamate uses have been cancelled or withdrawn. However, it is still necessary to recognize the signs of pesticide poisoning in animals and initiate prompt treatment when poisoning is suspected. When an animal presents any of the following symptoms for chlorinated hydrocarbons, organophosphates, carbamates, or pyrethroids it is essential that a history of the animal for 24 to 48 hours preceding onset of symptoms be compiled. It is also essential that pesticides in or around the area be located to determine the causative poison.

\section{Chlorinated Hydrocarbons}

Few chlorinated hydrocarbons are registered for use on or around animals. The chlorinated hydrocarbons are neurotoxins and usually act on the central nervous system, although kidney and liver functions can also be impaired. Symptoms of chlorinated hydrocarbon poisoning in animals are:

1. Vomiting

2. Restlessness or excitability

3. Tremor

4. Abnormal postures

5. Convulsions

6. Respiratory failure

7. Coma

When chlorinated hydrocarbon poisoning is suspected, a veterinarian should be consulted immediately. There are no specific antidotes, but therapy is directed towards removing the poison and treatment of the symptoms. If exposure is by dermal contamination, skin and hair should be washed with soap and water. Do not scrub or irritate the skin through brushing. If exposure is by ingestion, vomiting or gastric lavage is recommended (note: Do not induce vomiting if the ingested poison is principally a hydrocarbon solvent). Particularly in cattle, oral administration of activated charcoal (3 to 6 grams in a kilogram of water) will decrease absorption of the pesticide and mineral oil will help the animal eliminate the pesticide in the feces. Veterinarians may administer barbiturates in appropriate dosages if necessary to relieve the symptoms of restlessness or convulsions. Watch breathing closely, and provide artificial respiration or resuscitation if needed.

\section{Organophosphate Pesticides}

Organophosphate pesticides are neurotoxins that inhibit cholinesterase (irreversible). Specific antidotes are available for treating animals exhibiting organophosphate poisoning. Symptoms of organophosphate poisoning in animals are:

\section{Mild Poisoning}

\section{Sweating}

2. Tremors of tongue and eyelids

3. Excessive constriction of pupils of the eye

4. Tearing

5. Excessive salivation and other respiratory tract secretions

Moderate Poisoning

1.Earlier symptoms become more severe

2. Vomiting

3. Slow pulse

4. Uncontrollable muscle tremors

\section{Severe Poisoning}

1. Unconsciousness

2. Diarrhea

3. Pinpoint and non-reactive pupils

4. Loss of reflexes

5. Loss of sphincter control

6. Coma

7. Convulsions

8. Cyanosis

Animals poisoned with organophosphate pesticides should have prompt and aggressive therapy. For severe symptoms of organophosphate poisoning, veterinarians usually administer atropine to block the effect of the pesticide until atropinization (dryness of mouth and dilation of pupils) is reached. Repeated doses may be required every 5 to 10 minutes except in cattle where the effects of atropine generally last for 1 to 2 hours. Atropine should not be given to a cyanotic animal. After administering atropine, veterinarians also will administer intravenous doses of 2-PAM (Protopam chloride), to reactivate the cholinesterase, at 20 to $50 \mathrm{mg} / \mathrm{kg}$ 
body weight. If symptoms persist, a second dose of 2-PAM may be administered.

Morphine, aminophylline, and phenothiazine should not be given to animals exhibiting organophosphate poisoning.

Removal of the poison from the animal should be attempted as soon as possible. Hair and skin should be washed with soap and water if exposure was by dermal route. The skin should not be scrubbed or irritated. If the animal ingested the poison, induce vomiting within two hours or administer gastric lavage. Particularly in cattle, oral administration of activated charcoal ( 3 to 6 grams in a kilogram of water) decreases absorption of the pesticide and mineral oil helps the animal eliminate the poison in the feces. Watch breathing closely, and provide artificial respiration or resuscitation if needed. Despite attempted removal of the toxicant, the reappearance of signs of poisoning may persist for 24 to 48 hours in non-ruminants and up to 5 to 8 days in ruminants. It is suggested that close surveillance of affected animals be maintained for those time intervals.

\section{Carbamate Pesticides}

Carbamate pesticides are neurotoxins that inhibit cholinesterase (reversible). The symptoms of poisoning by carbamate pesticides are almost identical to the organophosphates. Treatment is also similar, except that 2-PAM should not be used because carbamate action on cholinesterase is reversible.

\section{Pyrethroid Pesticides}

Pyrethroid pesticides are synthetic derivatives of natural pyrethrins. They are neurotoxins that act on the sodium channels of insects and cause paralysis. Absorption through the skin of mammals is poor, so toxic reactions are rare. Mammalian toxicity to pyrethroid pesticides is extremely low, and chances for poisoning are almost nonexistent unless animals ingest formulations that have not yet been mixed for application. Early or mild symptoms of pyrethroid poisoning may be confused with organophophate or carbamate pesticide toxictity reactions. Symptoms of pyrethroid poisoning in animals are:

\section{Early/Mild Symptoms}
1. Salivation
2. Vomiting
3. Diarrhea
4. Mild tremors
5. Excitability/Depression

\section{Late/Severe Symptoms}

1. Hyperthermia

2. Hypothermia

3. Dyspnea

4. Severe tremors

5. Disorientation

6. Seizures

7. Death through respiratory failure

Pyrethroid poisoning is rare, and treatment for pyrethroid exposure is usually not required. In rare situations, for example, after ingestion of a dilute pyrethrin or pyrethroid formulation and if poisoning is suspected, a veterinarian should be contacted immediately. Following dermal exposure, the animal should be bathed with soap and water gently so that increased blood circulation and abrasion do not enhance absorption. Pyrethroids have been noted to cause eye irritation. Flush eyes with water and wash the area around the eyes with soap and water. Following ingestion, activated charcoal can be administered orally to increase elimination of pesticide if necessary. Seizures may need to be controlled with diazepam or methocarbamol.

\section{Selected References}

Berny, P., F. Caloni, S. Croubels, M. Sachana, V. Vandenbroucke, F. Davanzo and R. Guitart. (2010). Animal poisoning in Europe. Part 2: Companion animals. Veterinary Journal 183 (3): 255-259.

Khan C. M. and S. Line. (2005). The Merck veterinary manual, ninth edition. Merck, Whitehouse Station, NJ, U.S.A. Available online: http://www.merckvetmanual.org/ mvm/index.jsp (31 July 2012).

Raimon, G., S. Croubels, F, Caloni, M. Sachana, F. Davanzo, V. Vandenbrouke and P. Berny. (2010). Animal poisoning in Europe. Part 1: Farm livestock and poultry. Veterinary Journal 183 (3): 249-254.

Smith M. S. 2005. Applying pesticides correctly: a guide for private and commercial applicators. University of Kentucky Cooperative Extension Service, University of Kentucky, U.S.A. Available online: http://pest.ca.uky.edu/PSEP/Manuals/CORE-Manual.pdf (1 August 2012).

Walker, E. D., and J. A. Stachecki. 1994. Pest management for small animals: A training manual for commercial pesticide applicators and registered technicians (Category 7G). Michigan State University Extension, Michigan. Available online: http://www.pested.msu.edu/Resources/ bulletins/pdf/2492/E2492chap9.pdf (1 August 2012). 